\title{
Resolving the Calcium and Phosphorus Distribution in Casein Micelles in Bovine Milk: an in situ STEM/EDX Study as Applied to Hydrated Materials in Food Science
}

\author{
Brian R. Van Devener ${ }^{1}$, Almut H. Vollmer² and Nabil N. Youssef ${ }^{2}$ \\ 1. Nano-Scale Imaging and Surface Analysis Lab, University of Utah, Salt Lake City, UT, USA. \\ 2. Department of Nutrition, Dietetics and Food Sciences, Utah State University, Logan, UT, USA.
}

Casein micelles in bovine milk can generally be described as colloidal aggregates, on the order of 100 $\mathrm{nm}$ in diameter, which consist of casein proteins and associated minerals, primarily $\mathrm{Ca}$ and $\mathrm{P}$. It is through these micelles that the minerals, which are essential to neonatal development, are transported from mother to offspring in mammalian species [1]. In addition, caseins play a critical role in the nutritional and textural properties of dairy products such as cheese, liquid milk and yogurt [2]. Although there is a basic conceptualization of the structure of casein micelles, to date there has been no conclusive experimental evidence demonstrating the elemental distribution of $\mathrm{Ca}$ and $\mathrm{P}$ within the micelle.

Here, we present for the first time, results showing the spatial distribution of $\mathrm{Ca}$ and $\mathrm{P}$ in individual casein micelles. Utilizing a Protochips, Inc., Poseidon Select in situ liquid cell sample holder, we performed analytical scanning transmission electron microscopy (STEM) coupled with energy dispersive X-ray spectroscopy (EDX) on a JEOL JEM-2800 FEG S/TEM equipped with dual SDD EDX detectors. Figure 1 illustrates the configuration of the liquid cell. An EDX spectrum and spectral maps of the $\mathrm{Ca} \mathrm{K} \alpha$ and $\mathrm{P} \mathrm{K} \alpha \mathrm{X}$-ray edges within hydrated casein micelles, collected from a commercial milk sample, are presented in Figure 2. As seen here, both elements are co-located throughout the micelle ending the debate over the spatial distribution of $\mathrm{Ca}$ and $\mathrm{P}$. The calculated electron dose rate for this data set is $4.8 \mathrm{e}^{-} / \AA^{2}$ - less than the limit of $5 \mathrm{e}^{-/} / \AA^{2}$ which can cause damage to protein molecules [3]. The specific study of casein micelles herein also serves as the means to discuss the broader context of studying hydrated materials in their native state using analytical STEM.

There are significant challenges in analyzing the elemental composition of foods using STEM/EDX. TEM imaging of biological samples (including foods) typically involves adding image contrastenhancing agents in the form of metal salts containing heavy elements (e.g. Pb, U, Os). EDX spectra collected on materials with these elements have high spectral background and multiple X-ray edges from the staining agents, both of which may obscure spectral features of interest inherent within the sample under study. Further, the high electron beam currents typically needed to generate sufficient counts of X-rays from materials are potentially damaging to any radiation-sensitive materials, which includes those often studied in the food sciences [4]. The development of commercially available liquid cell holders, coupled with continual design improvements, has allowed researchers to image and measure dynamic processes of biological materials in native environments [5]. While recent advances in EDX detector configurations, including the development of silicon drift detectors (SDD) and multiple detector configurations, have improved X-ray count rates by orders of magnitude, applying EDX to liquid cell TEM presents new problems. Key issues are: line-of-sight between EDX detectors and samples in the liquid cells, significant background signal and $\mathrm{X}$-ray edges from $\mathrm{Si}$ and $\mathrm{N}$ in the chips, and $\mathrm{O}$ in aqueous media [6]. Further difficulties with conducting electron microscopy in an aqueous environment include negative effects of beam-mediated chemistry generated from reactive break-down products of water, such as $\mathrm{OH}^{-}$and $\mathrm{H}_{3} \mathrm{O}^{+}[7]$. 
This work represents, to our knowledge, the first in situ study of unstained, individual casein micelles utilizing STEM/EDX. Broader significance exists in the application of these techniques to studying the native micro and nano-scale structure of materials in the food sciences [8,9].

References:

[1] E Smyth et al., International Journal of Dairy Technology 57 (2004), p. 121.

[2] C de Kruif et al., Advances in Colloid and Interface Science 171-172 (2012), p. 36.

[3] R Glaeser, Structural Journal of Biology 128 (1999), p. 3.

[4] R Egerton, Ultramicroscopy 127 (2013), p. 100.

[5] A Demmert et al., in "Visualizing Macromolecules in Liquid at the Nanoscale", in Ross, F.M. (ed.)

Liquid Cell Electron Microscopy, (Cambridge University Press, Cambridge) p. 334.

[6] N Zaluzec et al., Microscopy and Microanalysis 20(2) (2014), p. 323.

[7] N Schneider et al., The Journal of Physical Chemistry C 118 (2014), p. 22373.

[8] The authors wish to acknowledge support from Leprino Foods Company for this work.

[9] This work made use of University of Utah USTAR shared facilities supported, in part, by the MRSEC Program of the NSF under Award Number DMR-1121252.

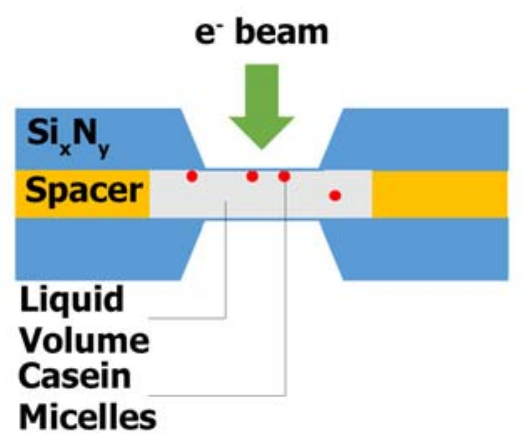

Figure 1. Liquid cell schematic. Two silicon nitride chips with 50-nm-thick (each) electron-beamtransparent windows are "sandwiched" together to form the hermetically sealed cell. The liquid thickness is set with 500-nm spacers between the chips, and the casein micelles are attached to the top window.

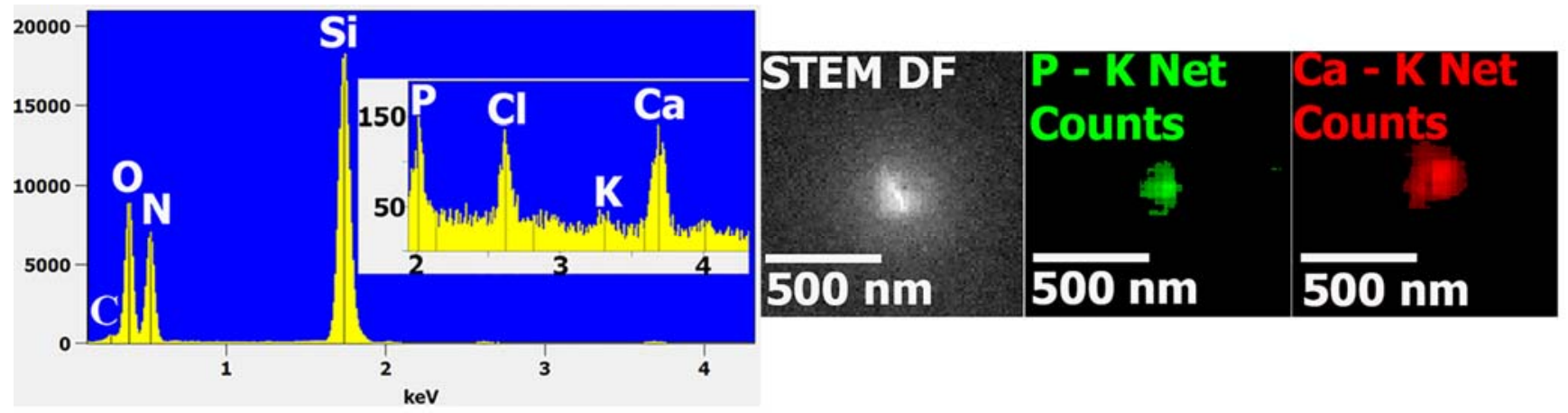

Figure 2. EDX spectrum (inset shows the region magnified between 2 and $4 \mathrm{keV}$ ) and backgroundsubtracted spectral maps of $\mathrm{P} \mathrm{K} \alpha$ and $\mathrm{Ca} \mathrm{K} \alpha \mathrm{X}$-ray edges within a single hydrated casein micelle. 